\title{
Perspektive Olympiaseelsorge
}

\section{Elisabeth Keilmann/Thomas Weber}

Die katholische Sport- und Olympiaseelsorgerin der Deutschen Bischofskonferenz sowie Geistliche Bundesbeirätin im DJK-Sportverband Elisabeth Keilmann und der evangelische Olympiapfarrer Thomas Weber sind für die seelsorgerische Betreuung der Olympiaathlet"innen, ibren Trainern und Betreuer*innen zuständig. Als Ansprechpartner für die Sportverbände ist es ibre Aufgabe, Sport und Kirche zu vereinen.

"Seelsorger - das ist ein passender Begriff. Es ist so gut, dass auch Trainer für die Seele dabei sind“, sagte eine Sportlerin einmal. Für sie sei es wichtig, dass bei großen Sportevents Seelsorgerinnen und Seelsorger dazu gehören, die nah bei den Menschen sind und als Ansprechpartner"innen für Gespräche über das Leben und den Glauben und für Begleitung zur Verfügung stehen.

Die Spitzensportseelsorge in Deutschland ist in der katholischen Kirche bei der Deutschen Bischofskonferenz (DBK) und in der evangelischen Kirche bei der Evangelischen Kirche Deutschlands (EKD) beheimatet. München 1972 prägte in besonderer Weise die Partnerschaft von Kirche und Sport. Weltoffen sollten diese Olympischen Spiele sein und für ein neues Verständnis von olympischer Kultur stehen. Allerdings wurde die Fröhlichkeit von dem Attentat auf die israelische Mannschaft überschattet. Im Ökumenischen Kirchenzentrum im Athletendorf fand schließlich eine Gedenkfeier für die Opfer statt und die kirchlichen Dienste gewannen an Bedeutung. Seit 1972 gehört das ökumenische Seelsorgeteam bei allen Olympischen Sommer- und Winterspielen, aber auch bei Sommeruniversiaden, den Weltspielen der Studierenden, als fester Bestandteil zur deutschen Mannschaft. Von 2006 an betreut auf evangelischer Seite Pfarrer Thomas Weber die deutsche Delegation und auf katholischer Seite seit 2018 als erste Frau Elisabeth Keilmann.

Neben dem Angebot von Gottesdiensten und der Unterstützung in Krisen ergibt sich so manches gute Gespräch, auch mit der Kirche Fernstehenden. Es geht nicht immer um „schneller, höher, stärker oder weiter“. Die wichtigsten Fragen im Sport haben mit dem Menschsein zu tun. Darin liegt der Grundgedanke der Sportseelsorge: für Menschen in unterschiedlichen Lebenssituationen ein offenes $\mathrm{Ohr}$ zu haben - nicht 
nur für die Sportler*innen, auch für Trainer*innen, Betreuer*innen oder Zuschauer*innen -, ihnen Mut zuzusprechen, zu trösten. Vorbereitende Begegnungen schaffen notwendige Kontakte. Und über die Großveranstaltungen hinaus ergeben sich durch die persönlichen Erfahrungen vielfältige Beziehungen.

Uschi Schmitz, Vize-Präsidentin des Deutschen Olympischen Sportbundes (DOSB) für den Bereich Leistungssport, führte kürzlich in einem Interview sehr zutreffend aus: „Bei den Olympischen Spielen sind seit vielen Jahren die beiden Sportpfarrer fester Bestandteil. Sie gehören genauso zum Team wie Physiotherapeuten, Ärzte und Trainer. Was ich geschätzt habe, ist, dass sie ganz verschiedene Aufgaben übernommen haben, keinem ein Gespräch aufzwingen, aber mit Vielen ins Gespräch gekommen sind. ... Es ist so eine Art Lebenshilfe bei Olympischen Spielen. Das Dasein ist das Wesentliche und dass die Athleten wissen, es gibt das Angebot. Das finde ich großartig!“ (in: srs-Magazin einSatz 4/2020, s. 34)

Das kirchliche Engagement bei Olympia hat auch einen theologischen Hintergrund. Im Sport erlebt der Mensch in besonderer Weise die Einheit von Körper, Seele und Geist und auch Kirche wendet sich dem ganzen Menschen zu. Im Neuen Testament verspricht Jesus: „Ich bin gekommen, damit sie das Leben haben und es in Fülle haben.“ (Johannesevangelium 10,10b). Das biblisch-ganzheitliche Menschenbild mit all den Erfahrungen von Freude, Gemeinschaft, Gelingen, Grenzen, Sieg und Niederlage bildet die Grundlage der Sportseelsorge. Es geht um das Aufgreifen von Fragen, die Menschen in vielfältigen Lebenskontexten betreffen.

Die Athletinnen und Athleten erfahren, dass sie aufeinander angewiesen sind, miteinander wetteifern und sich wechselseitig stärken können. Im Glauben wie im Sport geht es um eine gemeinsame Wertebasis, nämlich die Anerkennung der menschlichen Würde in der Person des Anderen. Ein Roll-Up-Banner von Team Deutschland in der deutschen Mannschaftsunterkunft in Südkorea 2018 bringt es auf den Punkt:

Wir leben Sport und lieben den Wettkampf.

Wir setzen uns keine Grenzen, aber klare Regeln.

Wir wollen Erfolge, aber nicht um jeden Preis.

Wir glauben an die verbindende Kraft des Sports.

Wir sind überzeugt, dass wir im Team am stärksten sind.

Wir sind stolze Botschafter für unser Land.

Wir schreiben Sportgeschichte für Deutschland.

Wir sind das Team Deutschland. 
Für alle, die Olympische und Paralympische Spiele hautnah aus den unterschiedlichsten Perspektiven miterleben können, für die Sportler*innen, Trainer*innen, Ärzt*innen, Betreuer*innen, Funktionär*innen, Medienvertreter"innen oder Fans, übt die Teilnahme eine Faszination aus. Spannende Wettkämpfe auf höchstem Niveau, begeistertes Publikum, neue persönliche Begegnungen, das Glück der Siegerinnen und Sieger und die Tränen der Unterlegenen prägen das Bild dieser Tage. Es ist greifbar, dass die Begeisterung für den Sport Menschen, Völker, Kulturen und Religionen vereint. Olympische Spiele und Paralympics haben die Kraft, Grenzen zu überwinden Türen zu öffnen, Werte zu erleben und Frieden zu stiften.

"Passion. Connected." - "Leidenschaft verbindet" - unter diesem Motto standen beispielsweise die Olympischen Winterspiele und die Paralympics 2018. Gerade in Südkorea erwärmte sich durch die Ausrichtung der Spiele die politische Eiszeit zwischen Nord- und Südkorea. Tauwetter setzte ein und die olympischen und paralympischen Tage standen ganz im Zeichen der Annäherung zwischen beiden Ländern.

Schon jede Eröffnungsfeier zu Beginn der Wettkämpfe liefert eindrückliche Bilder, wenn die teilnehmenden Nationen in das Stadion einziehen. $\mathrm{Da}$ folgen die Mannschaften aus dem Iran und Israel in alphabetischer Reihenfolge direkt aufeinander, um dem anschließenden Spektakel der Eröffnungszeremonie begeistert Seite an Seite sitzend zu folgen.

Dass die Leidenschaft verbindet, wird gerade auch an dem bunten Erscheinungsbild und dem Trubel des Olympischen Dorfes deutlich, dort wo die Athleten*innen und ihre Betreuer*innen während der Wettkampftage wohnen, essen und ihre Freizeit verbringen. Da begegnen sich Sportlerinnen und Sportler aus den verschiedensten Ländern. Und erst wenn der Schriftzug auf der Sportbekleidung zum Vorschein kommt, wird einem bewusst, dass viele aus Staaten stammen, die miteinander verfeindet sind oder in denen zu Hause Krieg und Waffengewalt herrschen.

Ein israelischer Trainer, der seinen Sohn betreute, erzählte am Rande eines Wettkampfes, dass sie ein solch freundliches und fröhliches internationales Miteinander zuvor selten erlebt hätten. Das bestätige ihm auch sein Sohn, der mit Sportlerinnen aus Malaysia ins Gespräch gekommen sei. Diese hätten sich gewundert, dass ein Israeli so sympathisch sein könne. Als er verwundert nachgefragt habe, hätten sie ihm ihre Reisepässe gezeigt: „Für alle Länder gültig, nur die Einreise nach Israel ist damit nicht erlaubt." 
Wie gut, dass es den Sport gibt! Dieser Gedanke bleibt nachhaltig im Gedächtnis.

Aber nicht nur das internationale Miteinander begeistert, sondern gerade auch der Teamgeist innerhalb der eigenen Mannschaft. Über alle Sportartgrenzen hinweg führen das Zusammenleben im Olympischen Dorf, das gegenseitige Anfeuern, das Interesse am Abschneiden der Anderen und die gemeinsame Freizeitgestaltung zu einem besonderen Wir-Gefühl. Es ist Gold wert und führt oft zu einer erstaunlichen Leistungssteigerung der Einzelnen. Um diese Erfahrung einmal mitzuerleben, sind selbst Top-Stars bereit, den Komfort des eigenen Hotelzimmers gegen die eher bescheidene Ausstattung einer Wohnung im Olympischen Dorf einzutauschen.

Für jede Athletin und jeden Athleten geht mit der Teilnahme an den Olympischen Spielen ein großer Traum in Erfüllung. Sie stellt den Höhepunkt der sportlichen Karriere dar. Was das bedeutet, führte einmal die Mutter einer Athletin mit Blick auf ihre Familiengeschichte aus. Sie berichtete, dass ihr Mann, der zugleich der Trainer der Tochter war, im Jahr zuvor verstorben sei. Die Tochter habe daraufhin ihre sportliche Laufbahn beenden wollen. Dann habe sie sich aber anders entschieden und weitergemacht. Tatsächlich sei es ihr gelungen, sich für die Olympischen Spiele zu qualifizieren, was ein toller Erfolg für die ganze Familie sei. Die unvergesslichen positiven Eindrücke, die sie hier in den Tagen sammeln könnten, würden sicherlich ihr ganzes weiteres Leben prägen. Das sei eine Entschädigung für viele, viele Jahre hartes Training im Hochleistungssport und die Zeit, das Geld und die ungezählten Kilometer, die sie als Familie in früheren Jahren für ihre Tochter geopfert hätten.

Die Hilfe bei der Verarbeitung von Sieg und Niederlage - nicht nur im Sport - gehört darum zu den zentralen Aspekten der kirchlichen Begleitung der Olympiamannschaft, die sozusagen „eine Gemeinde auf Zeit“ ist.

Ein sehr beeindruckender Moment bei Olympia war für mich (Thomas Weber) ein Gottesdienst an einem Sonntagnachmittag in der Wohnung einer Athletin, die am Vormittag Gold gewonnen hatte. Sie konnte ihr Glück kaum fassen, während andere Teilnehmerinnen in der Runde ihren Enttäuschungen über die eigene Leistung freien Lauf ließen und offen über ihre Gefühle sprachen. Solch „einen geschützten Raum“ ohne Kameras und Mikrofone anbieten zu können, darin besteht die große Chance der seelsorgerlichen Begleitung.

Mit dem Bild einer „Auszeit im Sport“ lassen sich ihr Selbstverständnis und ihre Wirkungsweise gut veranschaulichen. Der Begriff beschreibt die Unterbrechung eines Spiels, um einen Augenblick innezuhalten und auf eine Situation zu reagieren. Gerade das Religiöse Zentrum mitten 
im Olympischen Dorf bildet einen solchen Ruhepunkt in den hektischen Abläufen des sportlichen Großereignisses. Es ist von frühmorgens bis in die Abendstunden geöffnet. Dort gibt es Räume der Stille, es werden jedoch ebenso Gottesdienste, Messen, Gebete und angeleitete Meditationen für die Interessierten aus den verschiedenen Kulturen und Religionen angeboten, so wie es die Bestimmungen des IOC vorsehen.

Neben dem Angebot der Feier von Ökumenischen Gottesdiensten und Andachten im Religiösen Zentrum oder in ansprechenden Räumen in der deutschen Mannschaftsunterkunft bzw. im Deutschen Haus bieten die Seelsorger vor allem Möglichkeiten zum vertrauensvollen Gespräch an. Sie stehen als Ansprechpartner"innen nicht nur in Glaubensfragen zur Verfügung. Sie bringen viel "Zeit zum Hören" mit, sprechen Mut zu und trösten. Begegnungen ergeben sich mit den Mannschaftsmitgliedern, darüber hinaus aber auch mit Familienangehörigen der Sportler*innen, die zum Anfeuern dabei sind, mit ehemaligen Aktiven, mit Zuschauer*innen, Pressevertreter*innen, Politiker*innen usw.. In vielen Begegnungen wird interessanterweise deutlich, dass es eine große Schnittmenge zwischen Kirchengemeinden und Sportvereinen gibt. Die Einen aus der Familie engagieren sich im Sport und die Anderen sind in der Kirchengemeinde aktiv.

Das Anwachsen der öffentlichen Aufmerksamkeit, die Leistungsdichte und nicht zuletzt die Fragen der finanziellen Förderung stellen die im Sport Handelnden unter großen Erfolgsdruck. Zudem bildet der Sport nur einen Teilbereich unseres Lebens ab. Darum ist es ein wertvolles Gut und gerade bei Olympia etwas Nicht-Selbstverständliches, mit einem Seelsorger oder einer Seelsorgerin auch über ganz normale Dinge sprechen zu können, ohne Bedenken haben zu müssen, dass das Erzählte nachher nach außen getragen wird. Dies wissen viele zu schätzen.

In den Gesprächen mit den „Älteren“ werden so manches Mal die Höhen und Tiefen im Zusammenleben in Partnerschaft und Familie thematisiert. Die gemeinsame Zeit sei oft knapp.

Jüngere Sportlerinnen und Sportler bewegt oft der Blick in die Zukunft: „Wie geht es nach Olympia weiter? Wieviel bin ich bereit, in meinen Sport zu investieren?“ Aber auch die grundsätzlichen Fragen werden ausgesprochen: „Was wünsche ich mir für mein Leben? Was sind meine Ziele?"“

„Vor Jahren auf dem Flug nach Vancouver saß ich (Thomas Weber) neben einem Spieler, der zum Eishockeyteam gehörte. Über den Wolken erzählte er mir irgendwann die tragische Geschichte des am Gehirntumor erkrankten und später verstorbenen Mitspielers und Freundes Robert Müller. Es war meinem Sitznachbarn ein inneres Bedürfnis - und ich 
hörte zu. Am Ende sagte er dann: „Wenn so etwas passiert, dann merkt man, wie kostbar das Leben ist, und dann stellt sich doch jeder die Frage, was im Leben eigentlich wirklich zählt."

Gerade in Krisensituationen wird der Bedarf an seelsorgerlicher Begleitung besonders offenkundig. Dies zeigte sich etwa bei den Olympischen Spielen 2016 in Rio de Janeiro, als die Kompetenz der Seelsorger*innen in Krisenintervention und Trauerbegleitung gefordert war. Zur Halbzeit der Spiele ereignete sich nämlich ein tragischer Zwischenfall. Der 35-jährige Kanuslalomtrainer Stefan Henze verstarb an den Folgen seiner schweren Kopfverletzungen, die er sich wenige Tage zuvor bei einem Verkehrsunfall zugezogen hatte. In der Vergangenheit hatte es in der deutschen Mannschaft noch nie einen Todesfall während der Spiele gegeben. Die Anwesenheit der Seelsorger"innen als Notfallseelsorger"innen war nicht nur für die übrigen Teammitglieder, sondern ebenso für die Verantwortlichen des DOSB und des Auswärtigen Amts, die sich um die Familienangehörigen kümmerten, in diesen Tagen überaus wichtig. Unter dem Eindruck eines solch' schrecklichen Ereignisses kommen viele ins Nachdenken und stellen die Frage: Was ist denn eigentlich wirklich wichtig im Leben? Auch an der Gestaltung der sich anschließenden Gedenkfeier, die auf dem "Platz der Trauer" im Olympischen Dorf stattfand und an der 150 deutsche Teammitglieder sowie zahlreiche ausländische Weggefährt*innen des Verstorbenen teilnahmen, waren die Seelsorger*innen wesentlich mitbeteiligt.

Die Begleitung des Kanuslalom-Teams fand zwei Jahre später eine Fortsetzung, als sich der Todestag von Stefan Henze zum zweiten Mal jährte. Die Mannschaft bereitete sich gerade im Heimatort der Familie Henze auf die anstehende WM vor. Die Seelsorger*innen besuchten die Mannschaft im Trainingslager und im gemeinsamen Gespräch wurden die Geschehnisse von Rio und ihre Auswirkungen noch einmal aufgearbeitet. Es war ein tief beeindruckendes Erlebnis, als alle zum Abschluss mit den Angehörigen auf dem Friedhof das Grab von Stefan Henze besuchten und sich in einer kleinen Feier noch einmal an ihn erinnerten.

Neben der "Zeit im Gepäck" verteilen die Sportpfarrer ein weiteres Geschenk an die Sportlerinnen und Sportler - eine handliche selbstgestaltete Broschüre mit dem programmatischen Titel „Mittendrin“, die schon bei der Einkleidung im Vorfeld ausgelegt wird. Die Texte, Psalmen, Gebete und Meditationen in dieser Broschüre sollen helfen, in Momenten der Ruhe und Entspannung neue Kraft für die Seele zu schöpfen. 


\section{Kritische Begleitung}

Als Partner des Spitzensports bleibt Olympiaseelsorge aber nicht nur bei der Begleitung der Einzelnen und liturgischer Angebote stehen, sondern vertritt auf den unterschiedlichen Ebenen deutlich das christliche Menschenbild und damit die Sichtweise eines humanen Hochleistungssports: Sportlerinnen und Sportler sind als mündige Athlet"innen $\mathrm{zu}$ begreifen. Ihre persönliche Würde und Unversehrtheit sind gegen alle Vereinnahmungen durch strukturelle Zwänge zu verteidigen. Es darf nicht sein, dass Höchstleistungen forciert werden, dies aber zu Lasten der Gesundheit der Athlet"innen geht und von Veranstalter*innen, Geldgeber*innen, Regelwächter*innen oder auch Zuschauern billigend in Kauf genommen wird.

Es ist zudem offensichtlich, dass Kommerz, Gigantismus, Doping, Korruption und Umweltzerstörung die olympische Idee stark beschädigt haben. Für die jüngeren Athlet"innen mag zwar gelten, dass mit der Teilnahme und dem Sammeln von Erfahrungen das Ziel erreicht ist, aber Dabeisein allein scheint keineswegs alles zu sein, wenn in der öffentlichen Wahrnehmung nur die Medaillen gezählt werden und ein vierter Platz schon als Niederlage gilt.

Die Athlet*innen selbst laufen darum Gefahr, sich durch fremde, unerlaubte Mittel den Traum vom Sieg erfüllen zu wollen. Ebenso enden viele Karrieren aufgrund von Verletzungen oder Misserfolgen frühzeitig. Am Ende stehen junge Menschen ohne Ausbildung, Beruf und Perspektive da.

Auch Journalist"innen, Trainer*innen und andere im Sport verantwortlich Handelnde suchen das seelsorgerliche Gespräch - es geht zunehmend um berufsethische Fragen.

Im Hinblick auf zukünftige Entwicklungen wird ein neuer tragfähiger Wertekompass gewünscht. Gerade an diesem Punkt scheinen die kirchlichen Arbeitskreise Kirche und Sport gefragt zu sein, sich in die Diskussion mit den Verantwortlichen einzubringen.

\section{Außenwirkung und innerkirchliche Impulse}

Auf zwei weitere Aspekte der Olympiaseelsorge soll abschließend noch kurz hingewiesen werden:

Zum einen hat die Olympiaseelsorge eine enorme Außenwirkung. Dieser kirchliche Dienst im Sinne einer ökumenischen Gemeinschaft wird 
von sehr vielen positiv wahrgenommen und ist ein unverkennbares, sichtbares Zeichen für das Bemühen, ganz nah bei den Menschen zu sein. Oft heißt es sonst, dass sich die Kirchen aus immer mehr gesellschaftlichen Bereichen zurückzögen und damit ihren Einfluss aufgäben.

Die Seelsorger*innen geben ihren Kirchen ein Gesicht und nutzen zugleich die Chance, den christlichen Glauben weiterzugeben.

Gerade junge Menschen haben häufig in ihrer Lebensgeschichte einen immer geringeren Bezug zu biblischen Traditionen und Kirche. Andererseits machen sie sich Gedanken über ihr Leben und sind auf der Suche. So regt die Begegnung mit den Olympiaseelsorger*innen zu Neugierde und tiefer gehenden Fragen an.

Unter diesem Gesichtspunkt sind auch die Besuche der Seelsorger*innen bei den Teilnehmenden der Olympischen Jugendlager sehr wertvoll und werden gut angenommen. In Gesprächen, Diskussionen und Andachten berichten die Seelsorger*innen von ihrer Arbeit und haben zugleich die Möglichkeit, sich mit den jungen Erwachsenen über Themen wie "Werte im Sport“ oder „Frieden und Völkerverständigung“ auszutauschen.

Zum anderen werfen die gezielten Kontakte zum Spitzensport schließlich auch selbstkritische Fragen in Bezug auf die innerkirchliche Praxis auf. Gerade für junge Menschen ist es beispielsweise schwierig, kirchliche Aktivitäten mit dem im Leistungssport üblichen Trainings- und Wettkampfaufwand zu verbinden. So erzählt mancher Sportler stolz von seiner Konfirmation bzw. Firmung, erinnert sich gleichzeitig aber daran, dass dies damals gar nicht so einfach gewesen sei. Denn während die anderen Jugendlichen aus der Gruppe fleißig am Wochenende ihre Gottesdienstbesuche abhaken konnten, sei es für ihn als heranwachsenden Hochleistungssportler schwierig gewesen, den Erwartungen des Gemeindepfarrers zu entsprechen. Eine Athletin erinnerte sich schmunzelnd, dass sie im Vorfeld der Konfirmation alle sieben Heiligabend- und Weihnachtsgottesdienste in ihrer Gemeinde besucht habe, um auf die erforderliche Zahl der Gottesdienstanwesenheiten zu kommen.

Vielerorts wird in Gemeinden keine Rücksicht auf junge Spitzensportlerinnen und Spitzensportler genommen. An dieser Stelle sollte die Partnerschaft von Kirche und Sport nicht nur verbal betont werden, sondern auch praktische Vereinbarungen und Kompromisse im Gemeindeleben zur Folge haben. 
Beten Sie für die deutsche Mannschaft?

So manches Mal wird schließlich die Frage gestellt - nicht nur von Journalist"innen: „Beten Sie für die deutsche Mannschaft?" Unsere Antwort lautet dann: „Sie erwarten vielleicht, dass wir Nein sagen. Für Millionen von Menschen ist Sport ihr Ding. Und für uns auch. Ebenso gilt: Der Glaube ist uns so wichtig, dass wir keinen Bereich des Lebens davon ausklammern wollen. Weder den Alltag noch die Freizeit und somit auch den Sport nicht. Deshalb werden wir dafür beten, dass die deutschen Sportlerinnen und Sportler ihre beste Leistung abrufen können und ihre Wünsche und Hoffnungen auf sportliche Erfolge in Erfüllung gehen, so wie die Christ*innen in anderen Ländern auch für ihre Mannschaft beten. Aber wir werden bestimmt nicht dafür beten, dass die Deutschen mit allen Mitteln Siege erringen, sondern dass sie fair kämpfen, nach den geltenden Regeln handeln, und dass alle, die am Wettkampf teilnehmen, von Verletzungen verschont bleiben und die Fans fröhliche Begeisterung zeigen.“ 
